\title{
Search and characterization of T-type planetary mass candidates in the $\sigma$ Orionis cluster
}

\author{
K. Peña Ramírez ${ }^{1,2}$, M. R. Zapatero Osorio ${ }^{3}$, V. J. S. Béjarr ${ }^{1,2}$, R. Rebolo ${ }^{1,2,4}$, and G. Bihain ${ }^{5}$ \\ ${ }^{1}$ Instituto de Astrofísica de Canarias (IAC), 38205 La Laguna, Tenerife, Spain \\ e-mail: karla@iac.es \\ 2 Departamento de Astrofísica, Universidad de La Laguna, 38205 La Laguna, Tenerife, Spain \\ 3 Centro de Astrobiología (CSIC-INTA), 28850 Torrejón de Ardoz, Madrid, Spain \\ ${ }^{4}$ Consejo Superior de Investigaciones Científicas (CSIC), Madrid, Spain \\ 5 Leibniz Institute for Astrophysics Potsdam, An der Sternwarte 16, 14482 Potsdam, Germany
}

Received 2 March 2011 / Accepted 16 May 2011

\section{ABSTRACT}

\begin{abstract}
Context. The proper characterization of the least massive population of the young $\sigma$ Orionis star cluster is required to understand the form of the cluster mass function and its impact on our comprehension of the substellar formation processes. S Ori 70 (T5.5 \pm 1 ) and 73, two T-type cluster member candidates, are likely to have masses between 3 and $7 M_{\text {Jup }}$ if their age is 3 Myr. It awaits confirmation whether S Ori 73 has a methane atmosphere.

Aims. We aim to: i) confirm the presence of methane absorption in S Ori 73 by performing methane imaging; ii) study S Ori 70 and 73 cluster membership via photometric colors and accurate proper motion analysis; and iii) perform a new search to identify additional T-type $\sigma$ Orionis member candidates.

Methods. We obtained HAWK-I (VLT) $J, H$, and $\mathrm{CH}_{\text {uff }}$ photometry of an area of $119.15 \operatorname{arcmin}^{2}$ in $\sigma$ Orionis down to $J_{\text {comp }}=21.7$ and $H_{\text {comp }}=21$ mag. S Ori 70 and 73 are contained in the explored area. Near-infrared data were complemented with optical photometry using images acquired with OSIRIS (GTC) and VISTA as part of the VISTA Orion survey. Color-magnitude and color-color diagrams were constructed to characterize S Ori 70 and 73 photometrically, and to identify new objects with methane absorption and masses below $7 M_{\text {Jup }}$. We derived proper motions by comparing of the new HAWK-I and VISTA images with published near-infrared data taken $3.4-7.9$ yr ago.

Results. S Ori 73 has a red $H-C H_{40 f f}$ color indicating methane absorption in the $H$-band and a spectral type of T4 \pm 1 . S Ori 70 displays a redder methane color than S Ori 73 in agreement with its latter spectral classification. Our proper motion measurements $\left(\mu_{\alpha} \cos \delta=26.7 \pm 6.1, \mu_{\delta}=21.3 \pm 6.1\right.$ mas yr $^{-1}$ for S Ori 70, and $\mu_{\alpha} \cos \delta=46.7 \pm 4.9, \mu_{\delta}=-6.3 \pm 4.7$ mas yr $^{-1}$ for S Ori 73) are larger than the motion of $\sigma$ Orionis, rendering S Ori 70 and 73 cluster membership uncertain. From our survey, we identified one new photometric candidate with $J=21.69 \pm 0.12$ mag and methane color consistent with spectral type $\geq \mathrm{T} 8$.

Conclusions. S Ori 73 has colors similar to those of T3-T5 field dwarfs, which in addition to its high proper motion suggests that it is probably a field dwarf located at 170-200 pc. The origin of S Ori 70 remains unclear: it can be a field, foreground mid- to late-T free-floating dwarf with peculiar colors, or an orphan planet ejected through strong dynamical interactions from $\sigma$ Orionis or from a nearby star-forming region in Orion.
\end{abstract}

Key words. open clusters and associations: individual: $\sigma$ Orionis - stars: low-mass - brown dwarfs - techniques: photometric proper motions

\section{Introduction}

The discovery of isolated planetary mass objects (Lucas \& Roche 2000; Zapatero Osorio et al. 2000, 2002), i.e. free-floating substellar bodies with masses below the deuterium burning mass limit $\left(M \sim 12 M_{\text {Jup }}\right.$, Saumon et al. 1996; Spiegel et al. 2011), challenges our understanding of the substellar formation processes. The proper characterization of young planetary-mass objects and the analysis of their cluster membership in known star-forming regions are necessary for an accurate derivation of the substellar mass function. The $\sigma$ Orionis cluster, with an age of around 3 Myr (Sherry et al. 2008), low extinction (Béjar et al. 2004), solar metallicity (González Hernández et al. 2008), and location at a distance of 352 pc (Perryman et al. 1997), offers an excellent basis to carry out these studies (Béjar et al. 2001). Previous photometric searches identified two T-type member candidates, S Ori 70 (S Ori J053810.1-023626, Zapatero Osorio et al. 2002) and S Ori73 (S Ori J053814.5-024512,
Bihain et al. 2009). The former object has a spectral type T5.5 \pm 1 measured from its near-infrared spectrum covering the $H$ - and $K$-bands. Scholz \& Jayawardhana (2008) and Zapatero Osorio et al. (2008) reported that S Ori 70 has $J-K_{\mathrm{s}}$ and Spitzer colors redder than the typical colors of field $\mathrm{T}$ dwarfs, which are indicative of a low-gravity atmosphere. In contrast, the neutral $J-K_{\text {s }}$ and the mid-infrared colors of S Ori 73 do not appear to deviate significantly from the field $\mathrm{T}$ dwarf sequence. It awaits confirmation whether this object exhibits methane absorption in its atmosphere. Given its low brightness (S Ori 73 is about 1 mag fainter than S Ori 70 at near-infrared wavelengths), spectroscopy would require long integrations with large-size telescopes. Methane imaging (Rosenthal et al. 1996; Herbst et al. 1999; Tinney et al. 2005) provides a reasonable alternative means of analyzing the atmospheric properties of S Ori 73. The cluster membership confirmation of both S Ori 70 and 73 is relevant to assessing the form of the $\sigma$ Orionis mass function at planetary masses, since if both sources are true cluster members their masses are estimated to be 
in the interval 3-7 $M_{\text {Jup }}$. Additional T-type sources are identified in other stellar clusters and associations of different ages, such as the Pleiades (Casewell et al. 2007, 2011), the Trapezium (Lucas et al. 2006), IC 348 (Burgess et al. 2009), and $\rho$ Oph (Marsh et al. 2010; Alves de Oliveira et al. 2010).

Here we report on deep near-infrared and optical images collected with $8-10-\mathrm{m}$ class telescopes that will allow us to carry out a new "pencil" search for additional T-type cluster member candidates of $\sigma$ Orionis, to study the nature of S Ori 73, and to derive accurate proper motions of the two currently known $\mathrm{T}$ dwarf cluster member candidates. Our observations and data reduction are presented in Sect. 2, Section 3 presents the photometric and astrometric characterization of S Ori 70 and 73 along with their cluster membership assessment, and our new search is described in Sect. 4. Finally, we summarize our conclusions and final remarks in Sect. 5 .

\section{Observations}

\subsection{Near-infrared data}

Near-infrared images were collected with the wide-field camera HAWK-I (Pirard et al. 2004; Casali et al. 2006) installed at the Nasmyth A focus of unit 4 (Yepun) of the Very Large Telescope (VLT) on Cerro Paranal Observatory between September 2008 and March 2009. The on-sky field of view is $7.5 \times 7.5 \mathrm{arcmin}^{2}$ per single shot covered by four Hawaii $2048 \times 2048$ pixel detectors, which are separated by a cross-shaped gap of $15^{\prime \prime}$. The instrument pixel scale is 0 .' $^{\prime} 1064$. Our HAWK-I data consist of three different pointings, two of which are intended to image the known T-type cluster candidates S Ori 70 and 73 (located to the west of the central, bright, and multiple star $\sigma$ Ori); the third pointing is located about $20^{\prime} 56^{\prime \prime} 8$ to the south of the massive star. Figure 1 illustrates the three HAWK-I pointings. Images were taken using the broad-band filters $J$ and $H$, and the narrow-band filter $\mathrm{CH}_{4 \text { off }}$. The $\mathrm{CH}_{4 \text { off }}$ filter (central wavelength at $1.575 \mu \mathrm{m}$, width of $0.112 \mu \mathrm{m}$ ) covers wavelengths bluewards and out of the $H$-band strong methane absorption seen in T-type dwarfs (Burgasser et al. 2002; Geballe et al. 2002). A total of $4(J)$, $29(\mathrm{H}), 30\left(\mathrm{CH}_{4 \mathrm{off}}, \mathrm{S}\right.$ Ori 70$)$, and $60\left(\mathrm{CH}_{4 \mathrm{off}}, \mathrm{S}\right.$ Ori 73$)$ individual images were acquired at different (random) telescope offsets within a box of $15^{\prime \prime}$ in size for each pointing. This observing strategy allowed us to perform a proper Earth sky background contribution removal from the raw data. Images were obtained with typical seeing in the range 0.'34-0'.75; the field of S Ori 73 was observed with the best seeing. We provide the log of the observations in Table 1, where we include telescopes and instruments, central coordinates, field reference name (e.g., S Ori 70), observing filter, observing dates, total exposure time, seeing, completeness, and limiting magnitudes.

HAWK-I data were reduced using standard techniques for the near-infrared within the IRAF $^{1}$ environment including dark, flat field, pixel mask correction, and sky subtraction. Individual frames were aligned and stacked together to produce deep $J, H$, and $\mathrm{CH}_{4 \text { off }}$ images. By taking into account the gap between detectors and the offsets between individual images, we determined that the HAWK-I survey covers an area of $119.15 \mathrm{arcmin}^{2}$, $40.02 \operatorname{arcmin}^{2}$ of which was observed with a seeing of 0.34 in the $H$ band. The photometric analysis was performed by obtaining aperture and point spread function (PSF) photometry using routines of the DAOPHOT package. We defined the stellar

\footnotetext{
1 IRAF is distributed by National Optical Astronomy Observatories, which is operated by the Association of Universities for research in Astronomy, Inc., under contract to the National Science Foundation.
}

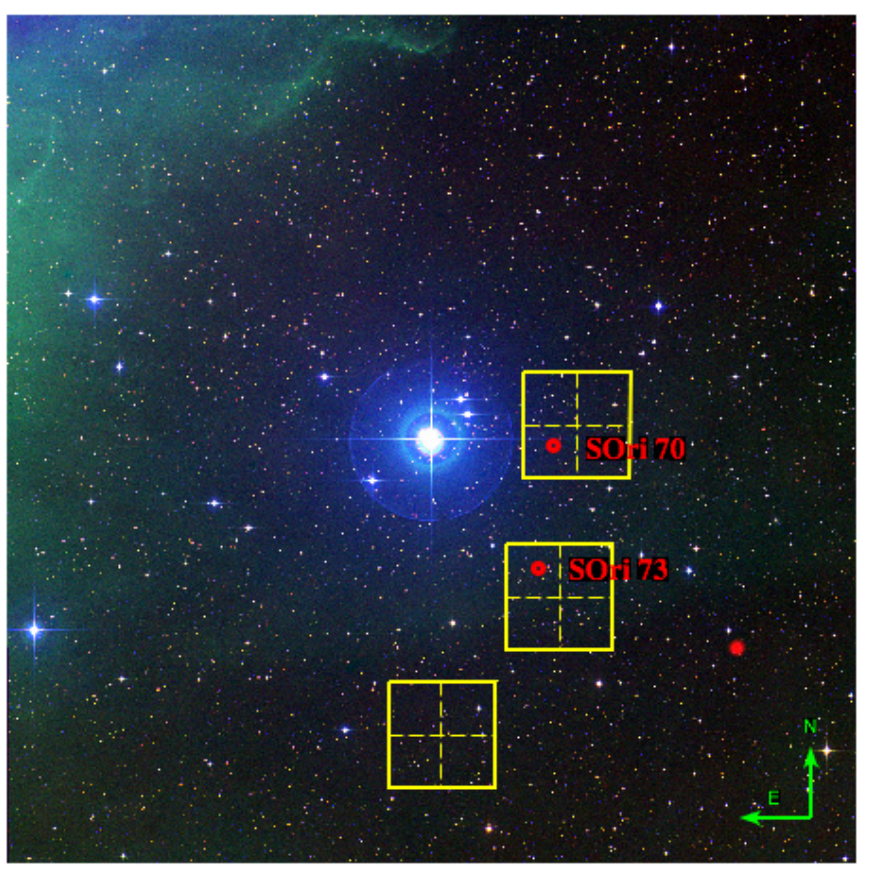

Fig. 1. False-color image of $1 \mathrm{deg}^{2}$ around the multiple, massive star $\sigma$ Orionis. This image was produced by combining the optical $B V R$ POSS-II data. The three pointings of the HAWK-I survey are plotted as yellow squares, each square showing the positions of the four detectors of the HAWK-I instrument. The locations of the $\sigma$ Orionis cluster T-type sources S Ori 70 and 73 are indicated.

PSF of our images by fitting Gaussian functions to 10-20 single stars per detector and pointing. The good seeing quality of the data $(\leq 0.75)$ allowed us to discriminate between resolved and unresolved (point-like) sources using the "sharpness" parameter provided by the DAOPHOT routines, which is defined as the difference between the square of the width of the object and the square of the width of PSF. Sharpness has values close to zero for single sources, large positive values for blended doubles and partially resolved galaxies and large negative values for cosmic rays and blemishes. Our adopted criterion flags an object detection with sharpness between -0.5 and +0.5 as a point-like source. As a precautionary measure, to ensure that we do not lose any potential interesting source, we also examined the photometry of object detections with sharpness values in the range 0.5-1.0 and unresolved (star-like) Gaussian full width at half maximum $(F W H M)$, as well as the photometry of faint sources within a radius of 5-6" from bright $(H \leq 19 \mathrm{mag})$ objects. Figure 2 displays the sharpness parameter for the S Ori 73 HAWK-I pointing; the vertical lines indicate our criterion for selecting unresolved sources. In Fig. 2, we also show the resulting fraction of resolved and unresolved object detections as a function of observed $H$-band magnitude. As expected, the number of extended (quite likely extragalactic) sources increases rapidly at faint magnitudes: at $H \sim 19 \mathrm{mag}$, about $50 \%$ of the objects appear resolved.

HAWK-I $J H$ instrumental magnitudes were converted into apparent magnitudes using the UKIRT Infrared Deep Sky Survey $\left(\right.$ UKIDSS) ${ }^{2}$ seventh data release (DR7). UKIDSS

2 The UKIDSS project is defined in Lawrence et al. (2007). UKIDSS uses the UKIRT Wide Field Camera (WFCAM; Casali et al. (2007)) and a photometric system described in Hewett et al. (2006). The pipeline processing and science archive are described in Irwin et al. (in prep.) and Hambly et al. (2008). 
Table 1. Log of optical and near infrared observations.

\begin{tabular}{|c|c|c|c|c|c|c|c|c|c|}
\hline Tel. & Instrument & \begin{tabular}{l}
\multicolumn{3}{c}{$\alpha(\mathrm{J} 2000) \delta$} \\
$\left(\begin{array}{lll}\mathrm{h} & \mathrm{m} & \mathrm{s}\end{array}\right)\left({ }^{\circ}, \quad, \quad\right)$
\end{tabular} & Field & Filter & Observing date & $\begin{array}{l}\text { Exposure } \\
\text { (s) }\end{array}$ & $\begin{array}{l}\text { Seeing } \\
(\operatorname{arcsec})\end{array}$ & $\begin{array}{c}\text { Compl. } \\
\text { (mag) }\end{array}$ & $\begin{array}{l}\text { Limit } \\
(\mathrm{mag})\end{array}$ \\
\hline GTC & $\begin{array}{l}\text { OSIRIS } \\
\text { OSIRIS }\end{array}$ & $\begin{array}{l}053826.8-024642 \\
053817.9-023708\end{array}$ & $\begin{array}{l}\text { S Ori } 73 \\
\text { S Ori } 70\end{array}$ & $\begin{array}{l}i^{\prime} \\
i^{\prime}\end{array}$ & $\begin{array}{l}2009 \text { Oct. 16, } 2010 \text { Jan. } 11 \\
2009 \text { Oct. } 13-15,2009 \text { Nov. } 19\end{array}$ & $\begin{array}{l}12636 \\
12506\end{array}$ & $\begin{array}{l}0.95 \\
0.90\end{array}$ & $\begin{array}{l}25.0 \\
25.0\end{array}$ & $\begin{array}{l}26.0 \\
26.0\end{array}$ \\
\hline VISTA & NIRCAM & $053820.6-024746$ & $\sigma$ Ori & $\begin{array}{l}Z \\
Y \\
J \\
H \\
K_{\mathrm{s}}\end{array}$ & $\begin{array}{l}2009 \text { Oct. } 20,21 \\
2009 \text { Oct. } 20 \\
2009 \text { Oct. } 19,20 \\
2009 \text { Oct. } 20 \\
2009 \text { Oct. } 20\end{array}$ & $\begin{array}{c}6084 \\
1008 \\
2112 \\
288 \\
288\end{array}$ & $\begin{array}{l}0.80 \\
0.90 \\
0.90 \\
0.90 \\
0.70\end{array}$ & $\begin{array}{l}22.6 \\
21.0 \\
21.4 \\
19.6 \\
18.6\end{array}$ & $\begin{array}{l}23.2 \\
21.4 \\
21.8 \\
20.0 \\
19.1\end{array}$ \\
\hline VLT & HAWK-I & $053808.5-024712$ & S Ori 73 & $\begin{array}{c}J \\
H \\
\mathrm{CH}_{4 \mathrm{off}}\end{array}$ & $\begin{array}{l}2008 \text { Sep. } 19 \\
2008 \text { Dec. } 8 \\
2009 \text { Feb. } 24,2009 \text { Mar. } 28\end{array}$ & $\begin{array}{c}160 \\
8410 \\
27000\end{array}$ & $\begin{array}{l}0.64 \\
0.34 \\
0.51\end{array}$ & $\begin{array}{l}22.5 \\
22.8 \\
22.2\end{array}$ & $\begin{array}{l}23.2 \\
23.5 \\
23.0\end{array}$ \\
\hline VLT & HAWK-I & $053803.3-023507$ & S Ori 70 & $\begin{array}{c}J \\
\mathrm{H} \\
\mathrm{CH}_{4 \mathrm{off}}\end{array}$ & $\begin{array}{l}2008 \text { Oct. } 27 \\
2009 \text { Mar. } 24 \\
2009 \text { Mar. } 16\end{array}$ & $\begin{array}{c}160 \\
8410 \\
13500\end{array}$ & $\begin{array}{l}0.75 \\
0.52 \\
0.57\end{array}$ & $\begin{array}{l}21.7 \\
21.5 \\
21.0\end{array}$ & $\begin{array}{l}22.2 \\
22.2 \\
21.8\end{array}$ \\
\hline VLT & HAWK-I & $053841.5-025657$ & & $\begin{array}{c}\mathrm{J} \\
\mathrm{H} \\
\mathrm{CH}_{4 \mathrm{off}}\end{array}$ & $\begin{array}{l}2009 \text { Feb. } 11 \\
2009 \text { Mar. } 30 \\
2009 \text { Mar. } 29\end{array}$ & $\begin{array}{c}160 \\
8410 \\
12615\end{array}$ & $\begin{array}{l}0.63 \\
0.75 \\
0.65\end{array}$ & $\begin{array}{l}22.0 \\
21.0 \\
20.5\end{array}$ & $\begin{array}{l}22.6 \\
21.5 \\
21.0\end{array}$ \\
\hline
\end{tabular}

sources with errors smaller than 0.1 mag were cross-correlated against our data. The dispersion in the photometric calibration is on average $0.04 \mathrm{mag}$; we added this uncertainty quadratically to the instrumental magnitude errors computed by IRAF for each source detection. For the $\mathrm{CH}_{4 \text { off }}$ filter, we obtained a photometric calibration relative to the $H$-band. Objects with $H$ magnitudes between 13 and 17 (probably Galactic stars of spectral types G$\mathrm{K}$ according to their UKIDSS colors) were forced to have a null $\mathrm{H}-\mathrm{CH}_{4 \text { off }}$ color. The typical dispersion in this procedure was $0.04-0.07 \mathrm{mag}$, which was added quadratically to the $\mathrm{H}-\mathrm{CH}_{4 \mathrm{off}}$ colors. In this way, we are able to recognize sources with an anomalous behavior in the $H$-band, i.e., sources with methane colors deviating from 0.0 . We note that for a given HAWK-I detector, the zero points are constant for the various pointings. However, the calibration shifts differ slightly (by up to $0.2 \mathrm{mag}$ ) from detector to detector.

We used additional near-infrared data in our study. As part of Visible and Infrared Survey Telescope for Astronomy (VISTA) science verification observations carried out between 2009 October 15 and November 02, the telescope VISTA (Dalton et al. 2006; Emerson et al. 2006) was used to explore an area of $\sim 30 \mathrm{deg}^{2}$ in the Orion Belt using the broad-band $Z Y J H K_{\mathrm{s}}$ filters (Petr-Gotzens et al., in prep.). This survey includes the $\sigma$ Orionis cluster. VISTA is equipped with an infrared camera as dedicated instrument with a pixel scale of 0.339 . In a single shot, VISTA provides on-sky coverage of $1.5 \times 1.0 \mathrm{deg}^{2}$. All VISTA science verification data were processed at the Cambridge Astronomy Survey Unit $(\mathrm{CASU})^{3}$ using the NIRCAM pipeline (version 0.9 .6 ). In our work, we employed version 2.1 of the photometric and astrometric VISTA Orion catalog, which provides magnitudes in all VISTA filters and right ascension and declination coordinates with a precision better than 0.11 . VISTA photometric and astrometric calibrations were performed by CASU using the 2MASS catalog (Skrutskie et al. 2006). Table 1 provides further instrumental and observing details of the VISTA Orion survey in the region of the $\sigma$ Orionis cluster.

Completeness and limiting magnitudes (see Table 1) of the HAWK-I and VISTA surveys in $\sigma$ Orionis were estimated from the distribution of the instrumental magnitude error bars as a

\footnotetext{
${ }^{3}$ http://casu.ast.cam.ac.uk
}

function of observed magnitudes (in a way similar to Bihain et al. 2009). We defined the completeness magnitude as the magnitude bin at which the average instrumental photometric uncertainty is $0.1 \mathrm{mag}$, which corresponds to a signal-to-noise ratio $(\mathrm{S} / \mathrm{N})$ close to ten. The limiting magnitude is defined to be the magnitude bin with an average photometric error of $0.25 \mathrm{mag}$ $(S / N \sim 4)$. We note that the S Ori 73 HAWK-I field, covering an area of $40.02 \mathrm{arcmin}^{2}$, was observed under very good seeing conditions $(0,34)$ and atmospheric transparency leading to rather deep images, which are about 1 mag fainter than the remaining HAWK-I data $\left(79.13 \operatorname{arcmin}^{2}\right)$. We also note that the VISTA Orion survey provides longer exposure times at $Z$ - and $J$ band for the $\sigma$ Ori region than for the rest of the Orion Belt area, therefore the resulting VISTA $\sigma$ Ori $Z$ and $J$ images are deeper.

\subsection{Optical data}

We obtained Sloan- $i^{\prime}$ images of the fields of S Ori70 and 73 using the optical OSIRIS instrument (Cepa et al. 2000) mounted on the Gran Telescopio de Canarias (GTC) at the Roque de los Muchachos Observatory between 2009 October and 2010 January. The camera consists of a mosaic of two 4096x 4096 pix $^{2}$ detectors with a pixel projection of 0 . $^{\prime} 125$ onto the sky; the OSIRIS field of view is $7.4 \times 7.4 \mathrm{arcmin}^{2}$ per single shot. A detector binning of $2 \times 2$ pixels was used. We obtained a total of 52 images with an integration time of $60.5 \mathrm{~s}$ and 156 images of $60 \mathrm{~s}$ for the field of S Ori 70; for the field of S Ori 73, a total of 52 images of $61.5 \mathrm{~s}$ and 156 images of $60.5 \mathrm{~s}$ were acquired. The total on-source exposure times were thus 3.47 and $3.51 \mathrm{~h}$ for the fields of S Ori 70 and 73, respectively. Raw frames were reduced using the CCDRED package within the IRAF environment. Individual frames were bias and dark current subtracted and flat-fielded. All frames were aligned, normalized to the same exposure time, and stacked together to produce deep $i^{\prime}$-band images overlapping with the S Ori 70 and 73 HAWK-I fields. The photometric analysis was performed using DAOPHOT routines, which includes the selection of objects with pointlike PSFs using the DAOFIND task (extended objects were mostly avoided) and aperture and PSF photometry. Instrumental 

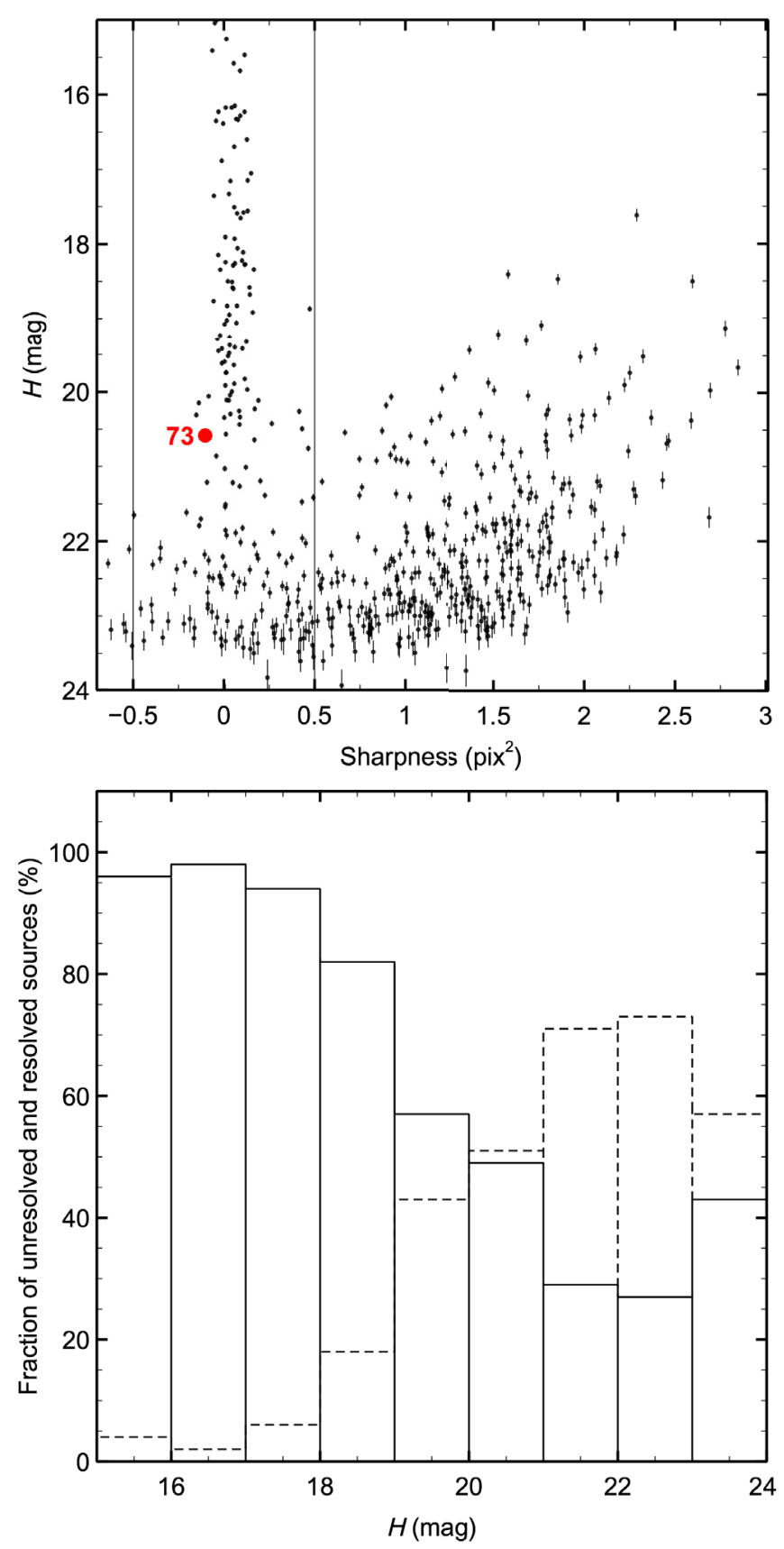

Fig. 2. Sharpness parameter (top panel) as a function of observed $H$-band magnitude for the HAWK-I S Ori 73 pointing, S Ori 73 plotted as a red filled circle. Our criterion for distinguishing resolved from unresolved sources is indicated with vertical lines: object detections between the two vertical lines are supposed to be unresolved (e.g., pointlike sources). The bottom panel illustrates the fraction of unresolved (solid line) and resolved (dashed line) sources per $H$-band magnitude bin.

magnitudes were transformed into observed magnitudes in the Sloan $i^{\prime}$ system with observations of photometric standard stars from Smith et al. (2002) obtained during the same nights in photometric sky conditions. Raw seeing ranged from 0 .' 7 to 1 ". 1 . The survey completeness and limiting magnitudes (as defined in the previous section) are $i^{\prime}=25$ and $26 \mathrm{mag}$, respectively. Table 1 summarizes the log of the OSIRIS observations.
Table 2. Optical and near infrared photometry for S Ori 70 and S Ori 73.

\begin{tabular}{lccc}
\hline \hline Instrument & Photometry & $\begin{array}{c}\text { S Ori 70 } \\
(\mathrm{mag})\end{array}$ & $\begin{array}{c}\text { S Ori 73 } \\
(\mathrm{mag})\end{array}$ \\
\hline OSIRIS & $i^{\prime}$ & $\geq 26.0$ & $\geq 26.0$ \\
NIRCAM & $Y$ & $20.85 \pm 0.13$ & $\geq 21.47$ \\
& $J$ & $19.84 \pm 0.06$ & $20.58 \pm 0.10$ \\
HAWK-I & $J$ & $19.85 \pm 0.05$ & $20.77 \pm 0.04$ \\
& $H$ & $20.10 \pm 0.05$ & $20.58 \pm 0.05$ \\
& $H-\mathrm{CH}_{4 \text { off }}$ & $0.61 \pm 0.07$ & $0.28 \pm 0.07$ \\
\hline
\end{tabular}

\section{Characterization of S Ori 70 and S Ori 73}

From our HAWK-I data, S Ori 73 remains unresolved at a spatial resolution of 0.34 (see Fig. 2), thus suggesting that it is a likely Galactic source. OSIRIS, HAWK-I, and VISTA photometry of S Ori 70 and 73 is given in Table 2. In VISTA, S Ori 70 is detected only in $Y$ and $J$ with the $Y-J$ color expected for its T5.5 spectral type. Both VISTA and HAWK-I magnitudes in the $J$-band agree within the photometric errors, and they are also close to the values reported in the literature (Zapatero Osorio et al. 2008), suggesting that S Ori 70's long-term photometric variability at around $1.2 \mu \mathrm{m}$ is below $0.15 \mathrm{mag}$.

In contrast, the $J$-band observations of S Ori 73 reported here are brighter by $0.14 \mathrm{mag}$ (HAWK-I) and $0.33 \mathrm{mag}$ (VISTA) than the value given by Bihain et al. (2009). S Ori 73 is detected only in the $J$ filter in the VISTA survey. In addition, the HAWK-I photometry in $H$-band of S Ori 73 is 0.25 mag brighter than the measurement provided in the discovery paper. These differences, which are slightly larger than the quoted photometric uncertainties ( $\sim 0.1 \mathrm{mag})$, may imply that the brightness of S Ori 73 varies. However, this requires confirmation using further data of higher accuracy.

Figure 3 displays the resulting color-magnitude diagrams of our HAWK-I data. Only unresolved sources are shown for clarity in the plots. The locations of objects such as S Ori 70 and 73 that are discussed in the subsequent sections are marked with different symbols and colors. We use Fig. 3 to study whether S Ori 73 has methane absorption in the $H$-band and to search for new $\sigma$ Ori T-type candidates in the cluster area of $119.15 \mathrm{arcmin}^{2}$ covered by HAWK-I. This area is twice as large as the one explored by Zapatero Osorio et al. (2002), and seven times smaller than the area surveyed by Bihain et al. (2009), which are two previous deep searches for $\mathrm{T}$ dwarfs in the cluster that yielded the findings of S Ori 70 and 73, respectively.

\subsection{Methane absorption}

One of our objectives is to confirm the presence of methane absorption in the atmosphere of the $\sigma$ Ori T-type photometric candidate S Ori 73, and constrain its spectral type. Given its low brightness, spectroscopy (even at near-infrared wavelengths) demands quite long integration times with current facilities. Tinney et al. (2005) and Goldman et al. (2010) showed that methane imaging can be very useful for characterizing $\mathrm{T}$ dwarfs since the methane color depends strongly on the T spectral subtypes (e.g., see Fig. 4 of Goldman et al. 2010). From Fig. 3, it becomes apparent that S Ori 70 and 73 are outliers. They have $\mathrm{H}-\mathrm{CH}_{4 \mathrm{off}}$ colors redder than point-like field sources of similar brightness, which we interpret as being caused by methane absorption in their atmospheres. These data thus confirm the "methane" nature of S Ori 73. The $\mathrm{H}-\mathrm{CH}_{4 \text { off }}$ color is expected to become redder for stronger methane absorption (i.e., increasing spectral types). 
K. Peña Ramírez et al.: Search and characterization of T-type planetary mass candidates in the $\sigma$ Orionis cluster
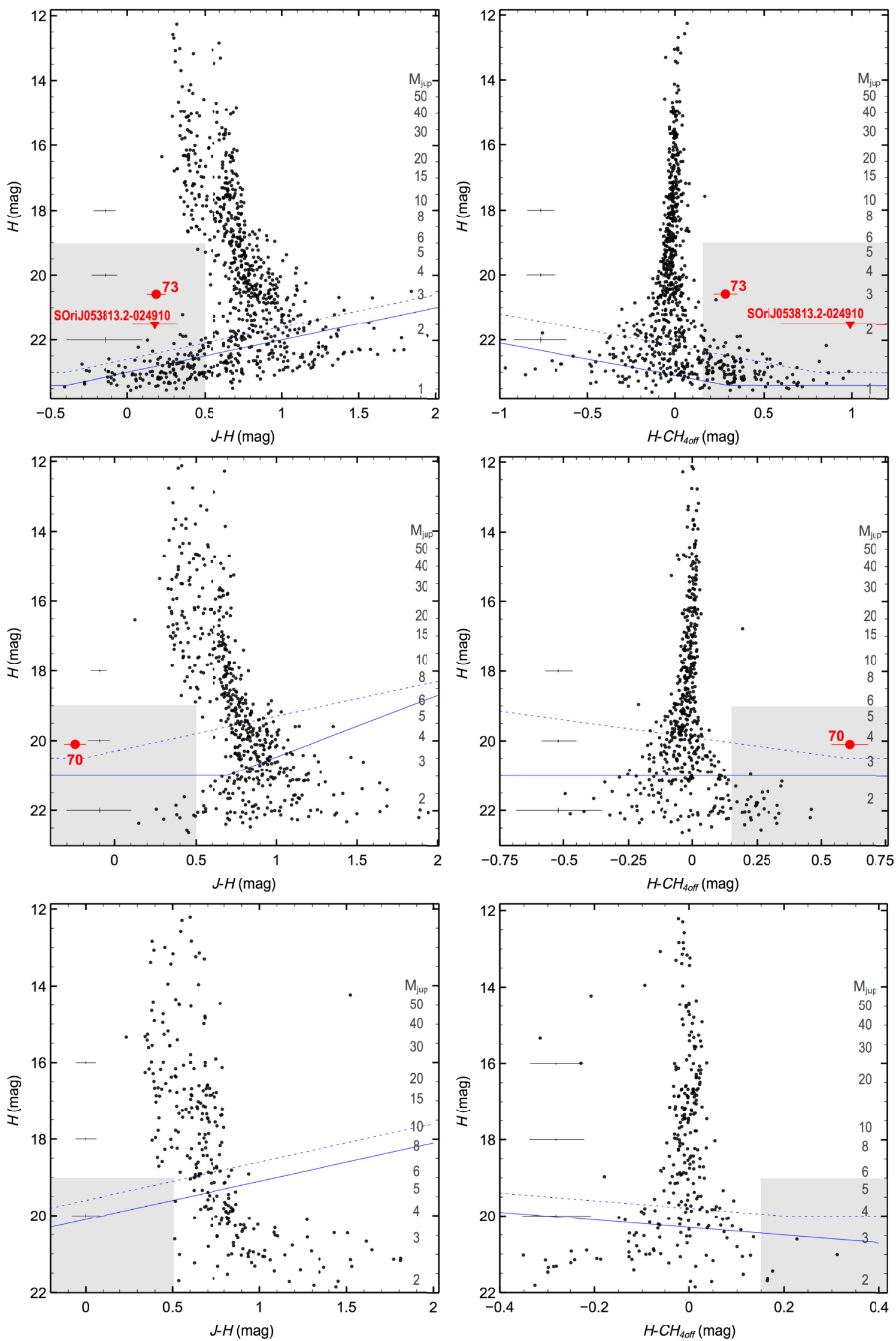

Fig. 3. Color-magnitude diagrams resulting from the HAWK-I survey. The top panels correspond to the S Ori 73 pointing, middle panels display the field around S Ori 70, and the lower panels illustrate the photometry of the third pointing. Sources discussed in this work are plotted as red circles (S Ori 70 and 73) and with a red triangle (new T-type candidate). Completeness and limiting magnitudes are indicated by a dashed and a solid line, respectively. Our photometric criteria to select new methane candidates are shown with a gray-shaded region. Average error bars are given to the left, and masses ( $M_{\mathrm{Jup}}$ ) predicted for the age of $3 \mathrm{Myr}$ are labelled on the right side of the diagrams. 
S Ori 73 has a $\mathrm{H}-\mathrm{CH}_{4 \text { off }}$ index bluer than S Ori 70 indicating less methane absorption intensity, i.e. earlier spectral type.

To estimate the spectral typing of S Ori 73, we used our HAWK-I data and a procedure similar to the one described by Goldman et al. (2010). The $H-\mathrm{CH}_{4 \mathrm{off}}$ color from HAWK-I needs to be calibrated against spectral type. For this, we retrieved near-infrared spectra of main-sequence stars and brown dwarfs of F- through L-types from the archives available in the literature (Rayner et al. 2009; Cushing et al. 2005) ${ }^{4}$. Most of these spectra were taken with the medium-resolution spectrograph SpeX at the NASA InfraRed Telescope Facility (IRTF) on Mauna Kea; they cover the spectral range of interest to ourselves $(\sim 1.3-$ $2.0 \mu \mathrm{m})$ and have a resolving power of $R \sim 2000$ and high $\mathrm{S} / \mathrm{N}$. To complete the spectral coverage towards cooler temperatures, spectra of field T-type dwarfs were retrieved from the libraries by Knapp et al. (2004), Chiu et al. (2006) and Golimowski et al. $(2004)^{5}$. These data, obtained with SpeX and the UKIRT Imaging Spectrometer and Cooled Grating Spectrometer, have a lower spectral resolution of $R \sim 150$. We use spectral types measured from optical data for the F-L sources, and from nearinfrared data based on the classification scheme of Burgasser et al. (2002) for the T objects. All these spectra were conveniently convolved with the HAWK-I $\mathrm{H}$ and $\mathrm{CH}_{4 \text { off }}$ filter transmission profiles and were flux integrated to derive the $\mathrm{H}-\mathrm{CH}_{4 \mathrm{off}}$ indices. The average color of the $\mathrm{G}-\mathrm{K}$ stars $(J-H \sim 0.4-$ $0.6 \mathrm{mag}$ ) was artificially set to 0.0 to keep the same photometric reference system as for the HAWK-I data (see Sect. 2.1). We estimated that the uncertainty in this procedure is \pm 0.1 mag in the methane color. We did not use the spectral library of T dwarfs by McLean et al. (2003) because the integration of the spectra systematically yielded very red methane colors that could not be reconciled with the values obtained from the other spectral libraries.

The resulting diagram displaying the $\mathrm{H}-\mathrm{CH}_{4 \mathrm{off}}$ values of field F-T sources as a function of the $J-H$ index is shown in Fig. 4. As expected, T ("methane") dwarfs are clearly recognized on the basis of their position in the diagram. Stars and L dwarfs have $\mathrm{H}-\mathrm{CH}_{4 \text { off }}$ colors between -0.1 and $\sim 0.03 \mathrm{mag}$, whereas substellar objects with types $\geq \mathrm{T} 3$ are characterized by $H-\mathrm{CH}_{4 \text { off }} \geq 0.1 \mathrm{mag}$ and $J-H$ typically below $0.5 \mathrm{mag}$. The location of S Ori 70 and 73 is indicated in Fig. 4. The methane color of S Ori 73 is consistent with a T4 spectral type, for which we estimate an uncertainty of one subtype. The photometric methane color of S Ori 70 resembles that of T $8 \pm 1$ field dwarfs. This spectral classification is marginally consistent and cooler than the one $(\mathrm{T} 5.5 \pm 1)$ from the literature (Zapatero Osorio et al. 2002). We also integrated the Keck spectrum of S Ori 70 (Zapatero Osorio et al. 2002) to derive its synthetic methane color; it is shown as an open circle in Fig.4. This computed color yields a classification of T6 (in agreement with the spectroscopic measurement); its associated error bar is rather large because the signal-to-noise ratio of the spectrum is poor. Both purely photometric and "integrated" measurements are consistent within the quoted uncertainties.

\subsection{Proper motions}

The HAWK-I and VISTA data add to the increasing battery of near-infrared images containing S Ori 70 and 73. The first images (including the discovery frames) available for these two

\footnotetext{
${ }^{4}$ http://irtfweb.ifa.hawaii.edu/spex/

IRTFSpectrallibrary/index.htm

${ }^{5}$ http://staff.gemini.edu/ sleggett/LTdata.html
}

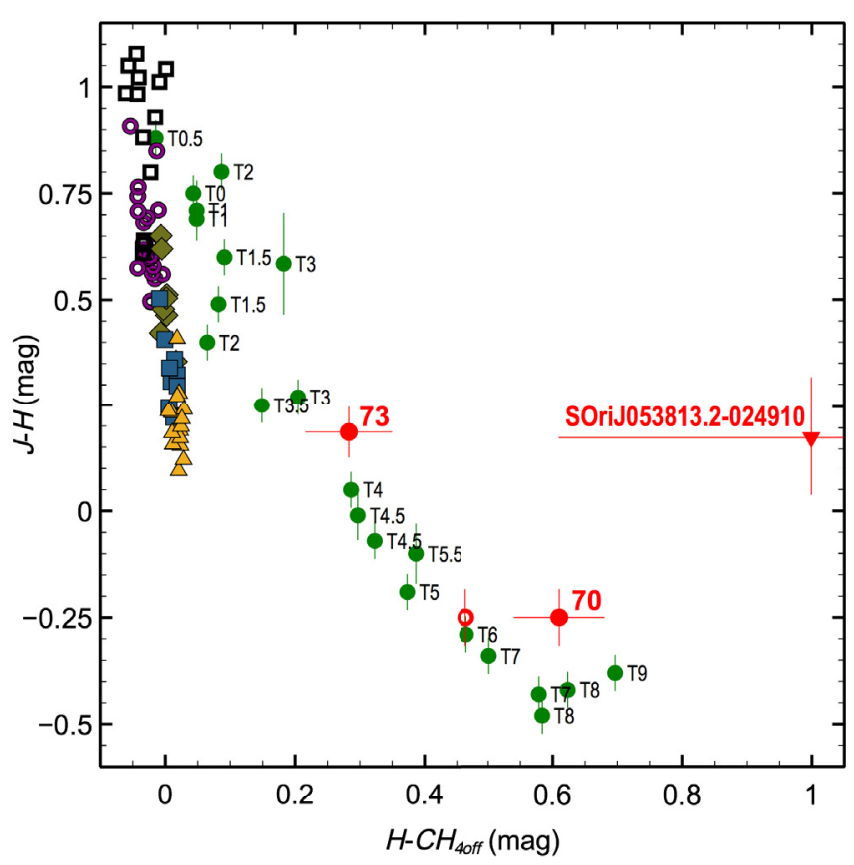

Fig. 4. Color-color diagram (including the HAWK-I methane color) for $\mathrm{T}$ (green), L (black), M (magenta), K (olive green), G (blue), and F (yellow) type field sources. The photometry of S Ori 70 and 73 is plotted as red filled circles and the new T-type candidate is shown with a red filled triangle. The red open circle corresponds to the synthetic methane color of S Ori 70 derived from the Keck spectrum published in the literature (see text).

T-type sources were obtained several years (3.4 to $\sim 8$ yr) before HAWK-I and VISTA observing runs, thus providing sufficient time baseline to carry out accurate proper motion measurements. The HAWK-I $H$-band and VISTA $J$-band images act as the second epoch data in our proper motion analysis. For S Ori 70, the first epoch frames are those presented in Zapatero Osorio et al. (2002, 2008): the $K_{\mathrm{s}}$ image obtained with the NIRC instrument on the Keck I telescope (2001 December 29, pixel size of 0!'15), and the $J$-band frame obtained with the Omega-2000 ( $\Omega 2000)$ instrument on the $3.5-\mathrm{m}$ telescope of the Calar Alto observatory (2005 October 22, pixel size of $0^{\prime}{ }^{\prime} 45$ ). The first epoch $J$-band image of S Ori 73 was taken with the ISAAC instrument on the VLT (2001 December 10, pixel size of 0.'15) presented in Caballero et al. (2007) and re-reduced in Bihain et al. (2009). The time separation between first and second epoch data is listed in Table 3 for each possible pair. Data of all epochs have good seeing quality, typically below $1^{\prime \prime}$, and both targets were detected with a $S / N$ greater than ten in all frames.

We determined the proper motions of S Ori 70 and 73 by comparing their $(x, y)$ pixel positions with respect to several tens of unresolved sources within a projected distance of 2-3 arcmin from the targets. We checked that an area of 4-9 $\operatorname{arcmin}^{2}$ provides a reasonable compromise between a high number (2050) of reference sources (employed to establish the astrometric transformations) and relatively "small" field distortions (introduced by the instrument optics and detector pixel-to-pixel differences). The only exception is the NIRC instrument (S Ori 70), which provides a rather small field of view $\left(32 \times 32 \operatorname{arcsec}^{2}\right)$ and a small number of reference sources (6). None of the reference sources is a known cluster member candidate; they are likely instead to be foreground and background objects. Pixel thirdorder polynomial transformations (second-order polynomials for NIRC) between epochs were calculated using the GEOMAP task 

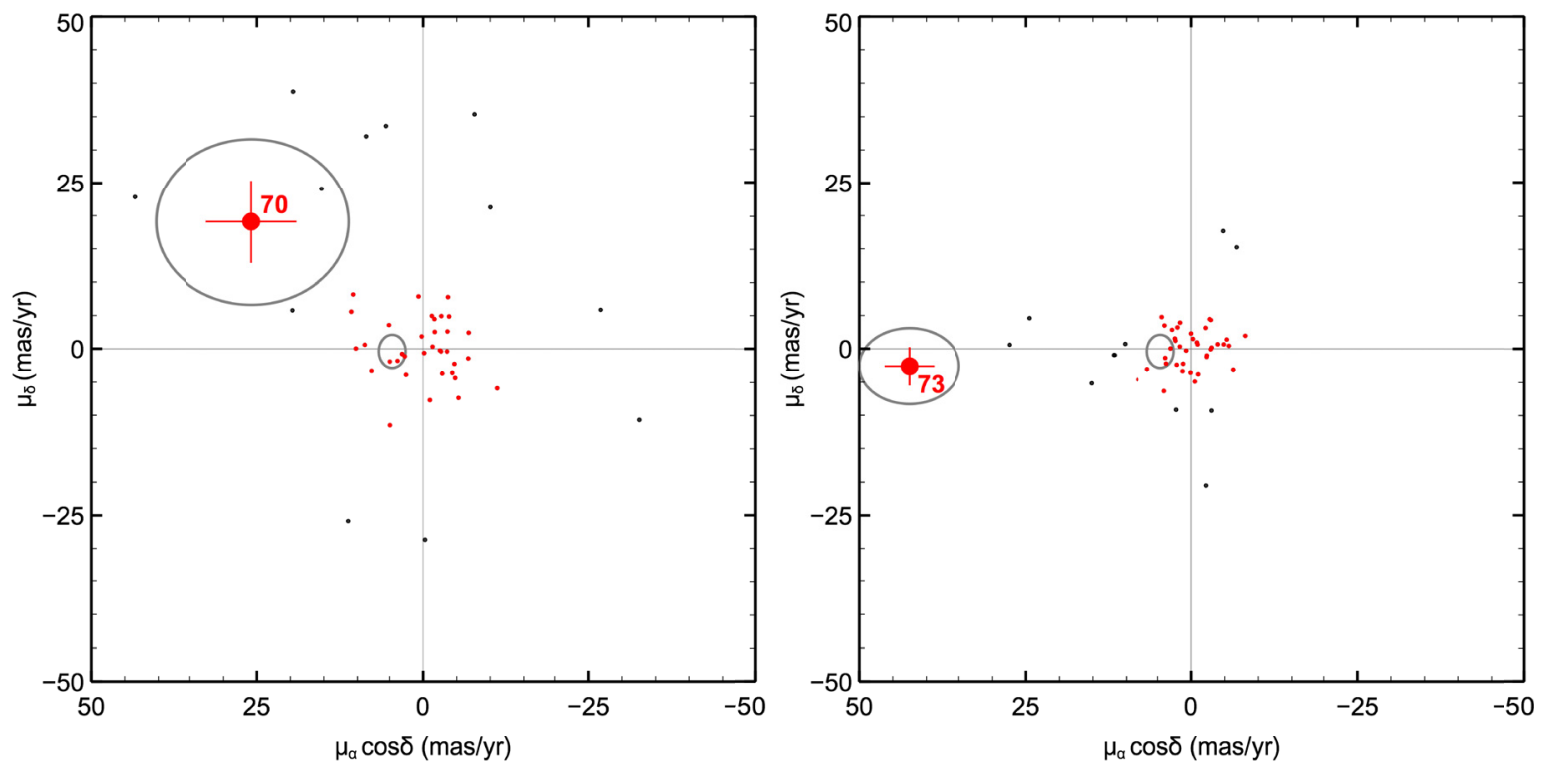

Fig. 5. Proper motion diagrams for S Ori 70 (left panel, $\Omega 2000 / H A W K-I$ data) and S Ori 73 (right panel, ISAAC/HAWK-I data). The two objects of interest are labelled. All identified sources within an area of $4 \mathrm{arcmin}^{2}$ around the targets are plotted as small dots. The sources used as astrometric references are shown with red tiny dots. The ellipses around S Ori 70 and 73 represent the 2- $\sigma$ proper motion uncertainties. The Hipparcos motion of the $\sigma$ Orionis cluster is depicted with a solid-line ellipse.

within the IRAF environment; the dispersion of the transformations was typically below 0.3 pix (or 0. '06) for both the $x$ and $y$ axes after rejecting reference sources that deviate by more than $3 \sigma$ from null motion, where $\sigma$ denotes the dispersion in the astrometric transformations. The resulting pixel shifts were converted into proper motions $\left(\mu_{\alpha} \cos \delta, \mu_{\delta}\right)$ by taking into account the time difference of the data, the pixel scale values, and the north-east orientation of the frames. Figure 5 illustrates the results of the proper motion derivation for S Ori 70 (Omega-2000 and HAWK-I data) and S Ori 73 (ISAAC and HAWK-I data). We provide our proper motion measurements in Table 3 . The proper motion uncertainties were calculated by adding quadratically the dispersion in the polynomial transformations and the errors in the targets centroids (0.03-0.12 pix). All individual determinations are consistent within the quoted uncertainties. In Table 3, we also determine the mean proper motions of S Ori 70 and 73 obtained by averaging individual measurements (excluding the measurement with the largest error bar for S Ori 70). We adopt these mean values in subsequent sections. Chromatic and differential refraction uncertainties do not contribute significantly to our proper motion measurements because we use near-infrared data and polynomial astrometric transformations of degree two and higher. Following the equations and discussion of Fritz et al. (2010), we estimate the chromatic and differential refraction errors to be below 1 mas.

\subsection{Cluster membership}

Both S Ori 70 and 73 present relatively distinct, large proper

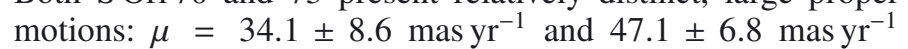
with position angles of $51.4 \pm 14 \mathrm{deg}$ and $97.7 \pm, 7 \mathrm{deg}$, respectively (position angles are measured east of north). Our measurement of $\mu$ for S Ori 70 is consistent at the level of 2.3- $\sigma$ with the proper motion value derived in Zapatero Osorio et al. (2008). Here and from now on, $\sigma$ represents the quadratic sum of the uncertainties associated with each proper motion component. The proper motion of S Ori 70 and 73 appear to be larger than the proper motion of the central, multiple star $\sigma$ Ori
Table 3. Proper motion measurements for S Ori 70 and S Ori 73.

\begin{tabular}{lcccc}
\hline \hline Object & Instruments & $\begin{array}{c}\Delta t \\
(\mathrm{yr})\end{array}$ & $\begin{array}{c}\mu_{\alpha} \cos \delta \\
\left(\mathrm{mas} \mathrm{yr}^{-1}\right)\end{array}$ & $\begin{array}{c}\mu_{\delta} \\
\left(\mathrm{mas} \mathrm{yr}^{-1}\right)\end{array}$ \\
\hline S Ori 70 & $\Omega 2000 / H A W K-I$ & 3.42 & $25.8 \pm 6.8$ & $19.1 \pm 6.3$ \\
& 22000/NIRCAM & 3.99 & $25.1 \pm 17.8$ & $8.1 \pm 11.9$ \\
& NIRC/HAWK-I & 7.23 & $25.5 \pm 5.2$ & $27.1 \pm 5.0$ \\
& NIRC/NIRCAM & 7.80 & $28.8 \pm 6.2$ & $17.7 \pm 6.9$ \\
& Average & & $26.7 \pm 6.1$ & $21.3 \pm 6.1$ \\
\hline S Ori 73 & ISAAC/HAWK-I & 6.99 & $42.5 \pm 3.7$ & $-2.6 \pm 2.9$ \\
& ISAAC/NIRCAM & 7.85 & $50.9 \pm 6.0$ & $-10.0 \pm 6.5$ \\
& Average & & $46.7 \pm 4.9$ & $-6.3 \pm 4.7$ \\
\hline
\end{tabular}

$\left(\mu=4.7 \pm 1.0\right.$ mas $\mathrm{yr}^{-1}$, position angle of $\left.95 \pm 10 \mathrm{deg}\right)$ as measured by Hipparcos (Perryman et al. 1997) by factors of $4.5-\sigma$ and 7.4- $\sigma$, respectively. These large differences are difficult to reconcile with the small (nearly null) motion of the $\sigma$ Orionis cluster.

At the distance of $\sigma$ Orionis (352 pc), the tangential velocities $\left(v_{\tan }\right)$ of S Ori 70 and 73 would be $57 \pm 14.3$ and $78.6 \pm 11.0 \mathrm{~km} \mathrm{~s}^{-1}$, respectively. These values are larger than those of brighter cluster members. S Ori 73 has a $v_{\text {tan }}$ in excess of the typical $v_{\tan }$ values of field $\mathrm{T}$ dwarfs published by Faherty et al. (2009), while S Ori 70 lies in the velocity range of known field dwarfs. If both sources were field objects, using their spectral classifications and observed $J$ magnitudes, they would be located at distances of 80-120 pc (S Ori 70) and 170$200 \mathrm{pc}$ (S Ori 73), translating into $v_{\tan }$ values of $13-19.5 \mathrm{~km} \mathrm{~s}^{-1}$ (S Ori 70) and 38-45 $\mathrm{km} \mathrm{s}^{-1}$ (S Ori 73). The $v_{\tan }$ of S Ori 73 then becomes consistent with the tangential velocities of field T dwarfs (Faherty et al. 2009), which have ages of a few Gyr, and the $v_{\tan }$ of S Ori 70 falls at the low values of the $v_{\tan }$ distribution of field T dwarfs.

Jeffries et al. (2006) and Sacco et al. (2008) showed that the radial velocity dispersion of $\sigma$ Orionis cluster members with an average mass between 0.1 and $0.5 M_{\odot}$ is $1.3 \mathrm{~km} \mathrm{~s}^{-1}$, which translates into a cluster internal proper motion dispersion of 
$0.8{\text { mas } \mathrm{yr}^{-1}}^{-1}(1-\sigma)$ using $352 \mathrm{pc}$ as the cluster distance. This velocity dispersion is a factor of $\sim 3$ smaller than the value found for the 1-Myr Orion Nebula cluster by Fúrész et al. (2008). Bihain et al. (2006) found that Pleiades substellar members with a mass of around 30-50 times the mass of Jupiter ( $\left.M_{\text {Jup }}\right)$ have a proper motion dispersion of $\geq 4$ mas $\mathrm{yr}^{-1}$, which is consistent with the linear relationship of equipartition of energy for a relaxed cluster. Given its total mass (between 150 and $250 M_{\odot}$, Hodapp et al. 2009), relatively low stellar density (Caballero 2008 ), and young age ( $3 \mathrm{Myr}$ ), the $\sigma$ Orionis cluster has likely existed for a few crossing times and as a consequence, the cluster may be dynamically unevolved. The expected relaxation time of $\sigma$ Orionis is around 21-35 $\mathrm{Myr}^{6}$, about ten times longer than the cluster age. By assuming that the cluster is close to relaxation, we estimate that the maximum proper motion dispersion expected for T-type members with a typical mass of $5 M_{\text {Jup }}$ is 5.5 mas yr$^{-1}(1-\sigma)$ or 11.0 mas yr$^{-1}(2-\sigma)$. The motions of both S Ori 70 and 73 appear to be beyond the conservative proper motion dispersion estimated for $\sigma$ Orionis planetary-mass objects.

The full extension of the $\sigma$ Orionis cluster is not properly determined yet, although various studies in the literature (e.g., Sherry et al. 2004; Lodieu et al. 2009; Béjar et al. 2011) have established that the majority of the cluster members $(\geq 80 \%)$ are located at distances of up to a radius of $0.5 \mathrm{deg}$ from the central O-type multiple star. At the current velocities of S Ori 70 and 73, these two objects would need only $\sim 1.0 \times 10^{5}$ and $\sim 0.8 \times 10^{5} \mathrm{yr}$ to cross the entire cluster, which represents less than $10 \%$ of the cluster age. The two sources might have recently acquired their large motions by means of strong dynamical interactions with more massive members of the cluster or as a result of expulsions from multiple, interacting systems. In addition, the motions (and large $v_{\tan }$ ) of S Ori 70 and 73 are incompatible with them being bound at large orbital separations from any cluster member in their surroundings, since their expected orbital velocities are necessarily smaller than the measured values. Their $v_{\tan }$ measurements also appear to be in excess of the cluster escape velocity at their position.

Because the $\sigma$ Orionis cluster belongs to the Orion starforming complex, occupying several tens of degrees on the sky, there is a possibility that either S Ori 70 or 73 is an ejected object from a nearby star-forming region. Over the past $12 \mathrm{Myr}$, Orion has given birth to at least ten thousand stars contained in sub-groups and short-lived clusters (e.g., Bally 2008). We linearly traced back the motions of S Ori 70 and 73 until they were beyond the limits of the Orion complex, and we searched for known star-forming regions that happen to lie along the projected motions. For S Ori 73, no region was identified. However, the backward projected motion of S Ori 70 ran at a separation of about $23^{\prime}$ from the LDN 1634 cometary molecular cloud about $6.0 \times 10^{5} \mathrm{yr}$ ago. This cloud, located west of the Orion Nebular Cloud, is probably shaped by the ultraviolet radiation from the Trapezium, and contains nine young stars (typically below $1 \mathrm{Myr}$ ) and three outflows, including the well-known HH 240/241 system studied by Bally et al. (2009). Lee et al. (2005) confirmed that LDN 1634 is currently undergoing stellar formation.

The photometric and spectroscopic properties of S Ori 70 and 73 can also give us additional information about their origins. The near- and mid-infrared colors of S Ori 73 do not deviate from those of field T3-T5 dwarfs, which contrasts with

\footnotetext{
${ }^{6}$ In Sherry et al. (2004), the relaxation time of a cluster similar to $\sigma$ Orionis was estimated to be seven times the crossing time $\left(t_{\text {cross }}\right)$, where $t_{\text {cross }} \leq 3-5 \mathrm{Myr}$.
}

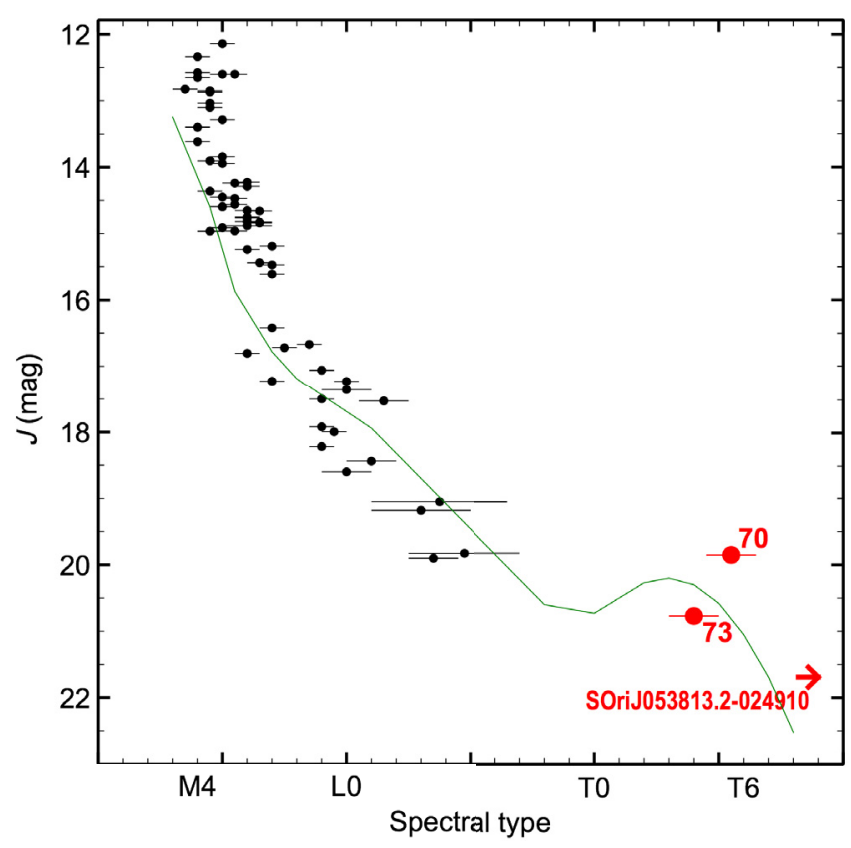

Fig. 6. Spectroscopic sequence of $\sigma$ Orionis cluster members (black dots). Data for mid-M to mid-L objects are taken from the literature (see Zapatero Osorio et al. (2008)). S Ori 70, 73 and the new T candidate are labelled. The average sequence of field M-, L-, and T-type dwarfs is normalized to the late-M types of the cluster and plotted as a green solid line. We use it to "predict" the location of $\sigma$ Orionis T-type members.

the colors of S Ori 70. As discussed in Zapatero Osorio et al. (2008), S Ori 70 displays red $J-K$ and Spitzer colors qualitatively in agreement with the theoretical predictions for solarmetallicity, low-gravity atmospheres. The reddish nature of this object was confirmed by Luhman et al. (2008), who found that it is slightly redder in [3.6]-[4.5] than the reddest field dwarfs. Scholz \& Jayawardhana (2008) argued that this property may be attributed to the presence of a surrounding dusty disk. This would support the youth of S Ori 70; we cannot rule out that it was formed in either $\sigma$ Orionis or a nearby Orion star-forming region such as LDN 1634. The colors of S Ori 73 suggest that it has a high-gravity atmosphere similar to field dwarfs of related spectral classification.

The spectral sequence of $\sigma$ Orionis is depicted in Fig. 6, where we include S Ori 70 and 73. All spectral types were derived from observed low-resolution spectra available in the literature, except for S Ori 73 whose spectral type is obtained from methane photometry. Overplotted onto the data is the average sequence of field M-, L-, and T-dwarfs conveniently normalized at the late-M types of the cluster. We use the normalized field sequence to aid the interpretation of the locations of S Ori 70 and 73 in Fig. 6 with respect to the positions of warmer $\sigma$ Orionis members. Despite of its cooler surface temperature, S Ori 70 is $0.5-1$ mag brighter than S Ori 73 at all wavelengths studied, which is not an expected situation if both objects are true cluster members (in principle, considering single sources, fainter objects should be cooler at a given age and metallicity). However, from Fig. 6 we cannot discard their membership in $\sigma$ Orionis individually: S Ori 70 and 73 appear slightly overluminous and underluminous than expected for T6- and T3-type members, respectively, yet their positions might be consistent with cluster membership if the $\sigma$ Orionis sequence has an intrinsic photometric dispersion of about $1 \mathrm{mag}$ at these faint magnitudes 
as suggested by the observed sequence delineated by $\mathrm{M}$ - and L-type members. This photometric dispersion may be caused by equal mass binaries, variability, slightly different ages, etc.

If neither S Ori 70 nor 73 were members of $\sigma$ Orionis but "old" field dwarfs, we would derive a T4-T6 volume density of $1.44 \pm 1.00 \times 10^{-2}$ dwarfs per $\mathrm{pc}^{3}$ by taking into account the area of $790 \mathrm{arcmin}^{2}$ and the magnitude interval $J=19-21.1 \mathrm{mag}$ explored by Bihain et al. (2009). This density is a factor of 2-10 higher than the average volume densities published in the literature for similar spectral types (Reylé et al. 2010; Metchev et al. 2008; Burningham et al. 2010), suggesting that either S Ori 70 or 73 is not a field object or that the density of field T dwarfs is higher in the direction towards the $\sigma$ Orionis cluster. If only one of the S Ori objects were a field dwarf, our volume density determination for T4-T6 sources would decrease to values in better agreement with the literature, and the cluster mass function at planetary masses would resemble that depicted in Fig. 13 of Bihain et al. (2009): the mass function appears to show a turnover below $6 M_{\text {Jup }}$. Cluster nonmembership of S Ori 70 and 73 would have a dramatic impact on the cluster mass function: the mass bin corresponding to the interval 3.5-6 $M_{\text {Jup }}$ would become "empty", suggesting an abrupt end to the formation of free-floating substellar objects. This finding and the mass at which it happens are critical parameters for the theory of stellar and substellar formation processes.

On the basis of all the preceding discussion, we conclude that S Ori 73 is probably not a member of the $\sigma$ Orionis cluster; it seems to be a field T3-T5 dwarf at a distance of 170-200 pc along the line-of-sight towards Orion. The origin of S Ori 70 remains unclear; it can be a field, foreground mid- to late-T freefloating dwarf with peculiar colors, or an orphan planet ejected from a nearby Orion star-forming region including $\sigma$ Orionis.

\section{New search for T-type cluster member candidates}

To identify new unresolved sources with methane absorption from the HAWK-I color-magnitude diagrams illustrated in Fig. 3, we applied the following photometric criteria: $J \geq 19$ mag, neutral near-infrared colors, i.e., $J-H \leq 0.5 \mathrm{mag}$, and red methane index, i.e., $H-\mathrm{CH}_{4 \mathrm{off}} \geq 0.15 \mathrm{mag}$. According to Fig. 4, the cut in the methane color allowed us to select sources with spectral types $\geq \mathrm{T} 3$. There are a total of nine sources, including S Ori 70 and 73, that fulfill our requirements. The seven new objects are found in the deepest images and have $H$ magnitudes and methane colors ranging from 20.8 to 23.0 mag and from 0.23 to $\sim 1.0 \mathrm{mag}$, respectively. Our survey is complete in the magnitude interval $J=19-21.7 \mathrm{mag}$. To complement the near-infrared data, we used the $Z$ (VISTA) and $i^{\prime}$ (OSIRIS) photometry. We note that neither S Ori 70 nor 73 are detected in the optical images. As observed from S Ori 70 and 73 and field T dwarfs, we expect T-type $\sigma$ Orionis member candidates to display a very red slope between the visible and near-infrared wavelengths. Consequently, we adopted additional photometric criteria for the newly selected candidates of $i^{\prime}-J \geq 5$ and $Z-J \geq 2.5$ mag. Five out of seven new candidates are clearly detected in the optical images suggesting that they have $i^{\prime} \leq 25$ and $Z \leq 23$ mag, thus are not T-type sources. A sixth candidate is not seen in the optical images, but is detected in the $K^{\prime}(2.12 \mu \mathrm{m})$ frames used in Bihain et al. (2009), which implies that $J-K^{\prime}=0.96 \mathrm{mag}$. This is not a typical color for $\mathrm{T}$ dwarfs, leading us to reject it as a methane candidate. Coordinates and photometry of the six rejected candidates are provided in Table 5. Five sources have brightness beyond the magnitude completeness of our survey. Finder charts are given in Fig. 7. We note that despite the presence of red methane colors, optical data are critical to the selection of reliable T-type candidates.

Only one object remains as a $\mathrm{T}$ dwarf cluster member candidate. It is listed in Table 4 and its finding chart is given in Fig. 7. The candidate lies within the magnitude completeness of our survey. It appears near a bright source that does not follow the $\sigma$ Orionis near-infrared photometric sequence based on its 2MASS data (Skrutskie et al. 2006). The candidate, S Ori J053813.2-024910, is located 2'.9 southwest of a bright object $(H=13.06 \pm 0.03 \mathrm{mag})$ and has a remarkably red $\mathrm{H}-\mathrm{CH}_{4 \text { off }}$ index; its associated photometric uncertainty is dominated by the large error bar of the $\mathrm{CH}_{4 \mathrm{off}}$-band photometry. We note that our optical data do not have the same spatial resolution as the near-infrared images, and that the wings of the bright sources prevent us from measuring deep $i^{\prime}$ and $Z$ magnitudes in the immediate surroundings of the stars. Therefore, we cannot conclude that S Ori J053813.2-024910 is a truly faint object complying with our photometric criteria in the red-optical wavelengths. Our visual inspection of the $\sigma$ Orionis Spitzer images (3.6, 4.5, 5.8, and $8.0 \mu \mathrm{m})$ obtained by Hernández et al. (2007) and Scholz \& Jayawardhana (2008) confirms that this source is not resolved from the bright star next to it.

If the new candidate turned out to be a true T-type object, we could estimate its spectral type using $J-H$ and $H-$ $\mathrm{CH}_{4 \text { off }}$ colors. The object is depicted in Fig. 4. From the position of S Ori J053813.2-024910 in the color-color diagram, we tentatively estimate a classification of $\geq \mathrm{T} 8 \pm 1$. The candidate has a very red methane color suggesting it might be an object cooler than the $\mathrm{T}$ dwarfs. Unfortunately, the $J-$ $H$ color of these kind of "ultracool" objects is not known. Reylé et al. (2010) provided volume densities for late-L and T-type dwarfs using astronomical observations of the CanadaFrance Brown Dwarf Survey. They quoted an object density of $1.4_{-0.2}^{+0.3} \times 10^{-3} \mathrm{pc}^{-3}$ for T0-T5.5, 5.3 $3_{-2.2}^{+3.1} \times 10^{-3} \mathrm{pc}^{-3}$ for T6 to T8 dwarfs and $8.3_{-5.1}^{+9.0} \times 10^{-3} \mathrm{pc}^{-3}$ for dwarfs cooler than T8. From these numbers and the magnitude of our candidate, we find that the contamination by field $\geq \mathrm{T} 8$ dwarfs in our survey covering $40.02 \operatorname{arcmin}^{2}$ (i.e., the deepest HAWK-I images where the candidate was found) is 0.08 objects $(19 \leq J \leq 23.2 \mathrm{mag})$. The estimated contamination by $\mathrm{T}$ dwarfs in the remaining HAWKI survey is $0.06(19 \leq J \leq 22.6 \mathrm{mag})$. Additional contamination by extragalactic sources may also be present in our survey; Goldman et al. (2010) discussed that quasars with redshifts between 1.30 and 1.48 have strong $\mathrm{H} \alpha$ emission and methane colors that mimic those of $\mathrm{T}$ dwarfs.

On the basis of the photometrically derived spectral type of S Ori J053813.2-024910, we can consider whether this candidate is a $\sigma$ Orionis member by studying its position in Fig. 6. The source deviates from the expected sequence. This discussion (and the one of previous section) is biased by the known properties of field $\mathrm{T}$ dwarfs, which have a mixture of ages and metallicities; very young $\mathrm{T}$ dwarfs with low-gravity atmospheres and same age and metal abundance might display a different behavior. If this candidate were confirmed as a $\sigma$ Orionis member using follow-up spectroscopic and astrometric data, its mass and surface temperature would be in the range 2-3 $M_{\text {Jup }}$ and $\leq 1300 \mathrm{~K}$.

\section{Conclusions and final remarks}

We acquired deep HAWK-I (VLT) $\mathrm{J}, \mathrm{H}$, and $\mathrm{CH}_{4 \text { off }}$ images covering an area of $119.15 \mathrm{arcmin}^{2}$ of the young $\sigma$ Orionis cluster. The completeness magnitudes of these observations are $J=21.7$ 


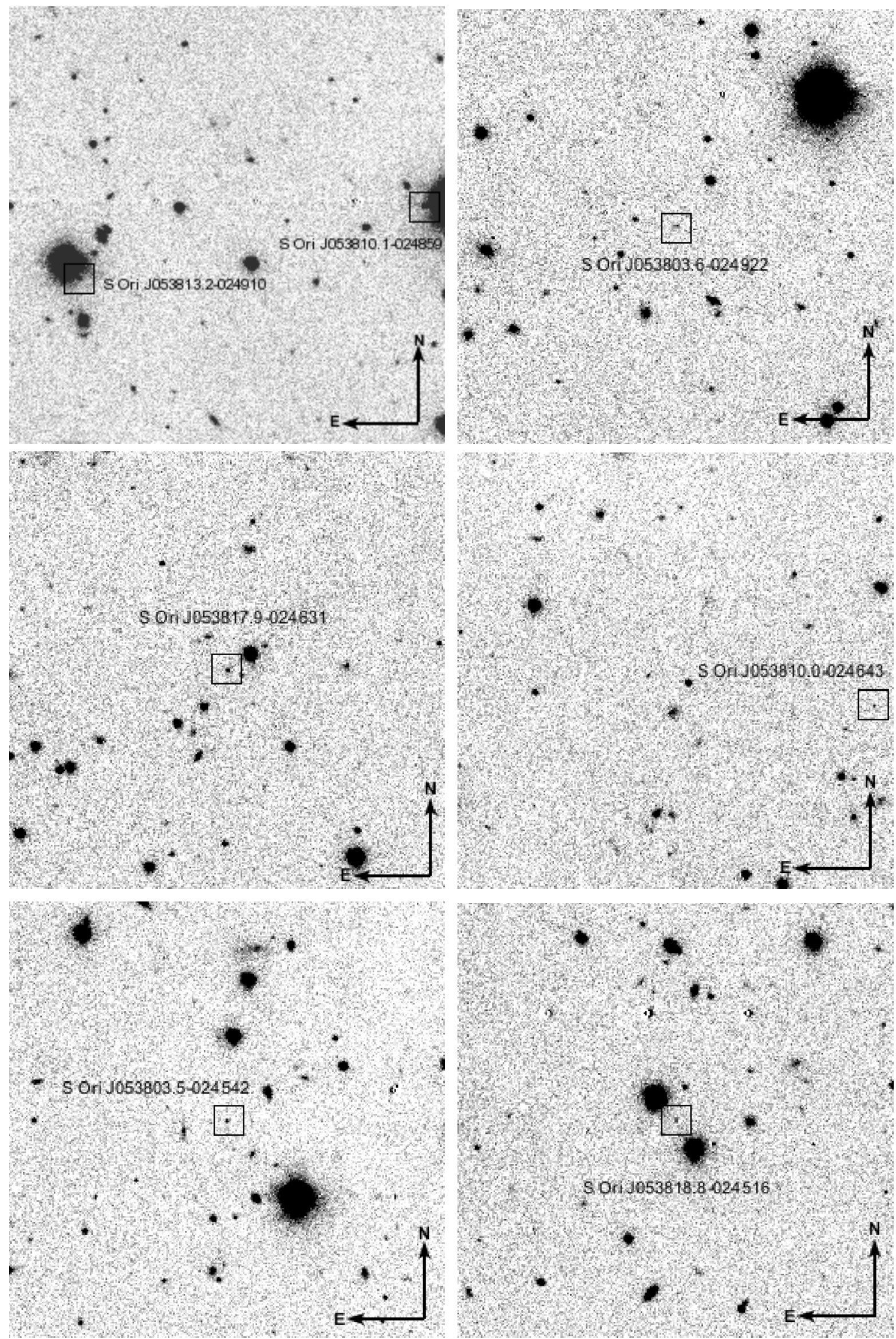

Fig. 7. Finding charts (HAWK-I $H$-band, $1 \times 1 \operatorname{arcmin}^{2}$ in size) for the new photometric T-type candidate (S Ori J053813.2-024910, upper left panel) and six rejected candidates. 
Table 4. Near infrared photometry for the new T-type candidate.

\begin{tabular}{lcccc}
\hline Candidate & $\begin{array}{c}J \\
(\mathrm{mag})\end{array}$ & $\begin{array}{c}H \\
(\mathrm{mag})\end{array}$ & $\begin{array}{c}J-H \\
(\mathrm{mag})\end{array}$ & $\begin{array}{c}H-\mathrm{CH}_{4 \text { off }} \\
(\mathrm{mag})\end{array}$ \\
\hline S Ori J053813.2-024910 & $21.69 \pm 0.12$ & $21.51 \pm 0.07$ & $0.18 \pm 0.13$ & $0.99 \pm 0.39$ \\
\hline
\end{tabular}

Table 5. Photometry of rejected candidates.

\begin{tabular}{lccccc}
\hline \hline Source & $\begin{array}{c}J \\
(\mathrm{mag})\end{array}$ & $\begin{array}{c}H \\
(\mathrm{mag})\end{array}$ & $\begin{array}{c}J-H \\
(\mathrm{mag})\end{array}$ & $\begin{array}{c}H-\mathrm{CH}_{4 \mathrm{off}} \\
(\mathrm{mag})\end{array}$ & $\begin{array}{c}i^{\prime}-J \\
(\mathrm{mag})\end{array}$ \\
\hline \hline S Ori J053803.6-024922 & $23.01 \pm 0.20$ & $22.59 \pm 0.10$ & $0.42 \pm 0.22$ & $0.37 \pm 0.16$ & $\leq 2.0$ \\
S Ori J053817.9-024631 & $22.80 \pm 0.18$ & $22.32 \pm 0.06$ & $0.48 \pm 0.19$ & $0.40 \pm 0.10$ & $\leq 2.2$ \\
S Ori J053810.0-024643 & $22.80 \pm 0.19$ & $22.60 \pm 0.08$ & $0.20 \pm 0.20$ & $0.54 \pm 0.13$ & $\leq 2.2$ \\
S Ori J053803.5-024542 & $22.74 \pm 0.18$ & $22.25 \pm 0.08$ & $0.49 \pm 0.20$ & $0.36 \pm 0.13$ & $\leq 2.3$ \\
S Ori J053810.1-024859 & $21.18 \pm 0.30$ & $20.76 \pm 0.04$ & $0.42 \pm 0.30$ & $0.23 \pm 0.07$ & $\leq 4.0$ \\
S Ori J053818.8-024516 & $22.86 \pm 0.19$ & $22.98 \pm 0.11$ & $-0.12 \pm 0.22$ & $0.98 \pm 0.14$ & $\geq 3.1^{a}$ \\
\hline
\end{tabular}

Notes. ${ }^{(a)}$ This rejected candidate has a $J-K^{\prime} \geq 0.5$ mag.

and $H=21 \mathrm{mag}$. The two T-type cluster member candidates previously reported in the literature, S Ori $70(\mathrm{~T} 5.5 \pm 1)$ and 73 , are included in the explored region. The "methane" filter is centered at $1.575 \mu \mathrm{m}$ and has a band width of $0.112 \mu \mathrm{m}$. It samples wavelengths bluewards of the $H$-band intense molecular methane absorption. The methane color $\mathrm{H}-\mathrm{CH}_{4 \text { off }}$ has a noticeable dependence on spectral type (or $J-H$ ), which we calibrated by using the 2MASS photometry of well known stars and brown dwarfs with classifications from $\mathrm{F}$ to late- $\mathrm{T}$ and by integrating their observed near-infrared spectra available in the literature. The HAWK-I data have allowed us to confirm for the first time the presence of methane absorption in the atmosphere of S Ori 73. Its $J-H$ and $H-C_{4 \text { off }}$ indices are consistent with a spectral type of T4 \pm 1 . Despite being about $0.5-1$ mag brighter than S Ori 73, S Ori 70 displays a redder $\mathrm{H}-\mathrm{CH}_{4 \text { off }}$ color compatible with its later spectral classification.

The new HAWK-I data and VISTA near-infrared images taken as part of the VISTA Orion survey were used to measure the proper motions of both S Ori 70 and 73 by comparison to the previous positions of the objects in near-infrared images collected 3.4-7.9 yr ago. We derived $\mu_{\alpha} \cos \delta=26.7 \pm 6.1$, $\mu_{\delta}=21.3 \pm 6.1 \mathrm{mas} \mathrm{yr}^{-1}$ for S Ori 70, and $\mu_{\alpha} \cos \delta=46.7 \pm 4.9$,

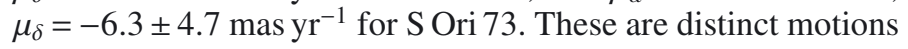
in excess of the Hipparcos $\sigma$ Orionis proper motion by $4.5-\sigma$ (S Ori 70) and 7.4- $\sigma$ (S Ori 73). We combined our knowledge of the color and astrometric properties of both $\mathrm{T}$ dwarfs to assess their cluster membership in $\sigma$ Orionis. S Ori 73 has near- and mid-infrared colors undistinguishable from old T3-T5 dwarfs in the field and a large proper motion deviating by $7.4 \sigma$ from that of the cluster, suggesting that it is probably a field dwarf situated at a distance of 170-200 pc. As reported in the literature, S Ori 70 displays near- and mid-infrared colors redder than typical field $\mathrm{T}$ dwarfs, a feature that is qualitatively predicted for low-gravity atmospheres according to state-of-the-art models (see Zapatero Osorio et al. 2008). We traced back the projected proper motion of S Ori 70 and found that it likely ran close to LDN 1634 about $6.0 \times 10^{5}$ yr ago. LDN 1634 is a nearby Orion star-forming region located to the southeast of $\sigma$ Orionis. We conclude that the origin of S Ori 70 remains unclear: it can be a field, foreground mid- to late-T free-floating dwarf with peculiar colors, or an orphan planet ejected through strong dynamical interactions from a nearby Orion star-forming region including $\sigma$ Orionis. If S Ori 70 and 73 were field T4-T6 dwarfs, we would determine an object volume density a factor of 2-10 higher than the values of the literature, and the impact on the $\sigma$ Orionis planetary mass function would be dramatic suggesting a decrease in cluster members with planetary masses in the interval 3.5-6 $M_{\text {Jup }}$.

We also carried out a photometric search for additional T-type cluster member candidates with masses in the interval 2 $7 M_{\text {Jup }}$ by combining the HAWK-I data with images collected with OSIRIS ( $i^{\prime}$-band) and the VISTA images ( $Z$-band). The search is complete in the magnitude interval $J=19-21.7 \mathrm{mag}$. We applied the following photometric criteria $J \geq 19, J-H \leq 0.5$, $H-C H_{4 \text { off }} \geq 0.15, i^{\prime}-J \geq 5$, and $Z-J \geq 2.5 \mathrm{mag}$, which allowed us to identify candidates with likely spectral types $\geq \mathrm{T} 3$. We note that despite the presence of red methane colors, optical data are crutial for selecting reliable T-type candidates because these objects are characterized by extremely red opticalto-near-infrared indices as opposed to (extragalactic) sources that might also show red methane colors. Only one object with $J=21.69 \pm 0.12 \mathrm{mag}$ fulfilled our requirements. However, it lies within a few arcsec of a bright source, which has prevented us from studying whether this candidate has genuinely a very red slope from the $i^{\prime}$ wavelengths to the $J$-band. Its $\mathrm{CH}_{4 \text { off }}$ color suggests that it might have a spectral type $\geq \mathrm{T} 8$. Additional photometry and spectroscopy are highly required to assess the true nature of this candidate.

Acknowledgements. We thank E. L. Martín and the anonymous referee for constructive discussion on proper motions. We also thank T. Mahoney (IAC) for the English edition of this manuscript. Based on observations made with ESO Telescopes at the Cerro Paranal Observatory under program ID 382.C-0115, and observations made with the Gran Telescopio de Canarias (GTC) operated on the island of La Palma in the Spanish Observatorio del Roque de los Muchachos of the Instituto de Astrofísica de Canarias. We used the Second Palomar Observatory Sky Survey (POSS-II), which was made by the California Institute of Technology with funds from the National Science Foundation, the National Aeronautics and Space Administration, the National Geographic Society, the Sloan Foundation, the Samuel Oschin Foundation, and the Eastman Kodak Corporation. The Oschin Schmidt Telescope is operated by the California Institute of Technology and Palomar Observatory. This research has been supported by the Spanish Ministry of Science and Innovation (MICINN) under the projects AYA2010-21308-C03-01, AYA2010-21308-C03-02, AYA2010-20535 and CSD2006-00070.

\section{References}

Alves de Oliveira, C., Moraux, E., Bouvier, J., et al. 2010, A\&A, 515, A75 Bally, J. 2008, in Handbook of Star Forming Regions, ed. B. Reipurth, ASP Monograph Publication, 4, 24 [arXiv: 0812 . 0046] 
Bally, J., Walawender, J., Reipurth, B. \& Megeath, S. T. 2009, AJ, 137, 3843 Béjar, V. J. S., Martín, E. L., Zapatero Osorio, M. R., et al. 2001, ApJ, 556, 830 Béjar, V. J. S., Zapatero Osorio, M. R., \& Rebolo, R. 2004, Astron. Nachr., 325, 705

Béjar, V. J. S., Rebolo, R., Zapatero Osorio, M. R., et al. 2011, ApJ, submitted Bihain, G., Rebolo, R., Béjar, V. J. S., et al. 2006, A\&A, 458, 805

Bihain, G., Rebolo, R., Zapatero Osorio, M. R., et al. 2009, A\&A, 506, 1169 Burgasser, A. J., Kirkpatrick, J. D., Brown, M. E., et al. 2002, ApJ, 564, 421 Burgess, A. S. M., Moraux, E., Bouvier, J., et al. 2009, A\&A, 508, 823 Burningham, B., Pinfield, D. J., Lucas, P. W., et al. 2010, MNRAS, 406, 1885 Caballero, J. A. 2008, MNRAS, 383, 375

Caballero, J. A., Béjar, V. J. S., Rebolo, R., et al. 2007, A\&A, 470, 903

Casali, M., Pirard, J., Kissler-Patig, M., et al. 2006, in SPIE Conf., 6269

Casali, M., Adamson, A., Alves de Oliveira, C., et al. 2007, A\&A, 467, 777

Casewell, S. L., Dobbie, P. D., Hodgkin, S. T., et al. 2007, MNRAS, 378, 1131

Casewell, S. L., Jameson, R. F., Burleigh, M. R., et al. 2011, MNRAS, 412, 2071

Cepa, J., Aguiar, M., Escalera, V. G., et al. 2000, in SPIE Conf. 4008, ed. M. Iye, \& A. F. Moorwood, 623

Chiu, K., Fan, X., Leggett, S. K., et al. 2006, AJ, 131, 2722

Cushing, M. C., Rayner, J. T., \& Vacca, W. D. 2005, ApJ, 623, 1115

Dalton, G. B., Caldwell, M., Ward, A. K., et al. 2006, in SPIE Conf. 6269

Emerson, J., McPherson, A., \& Sutherland, W. 2006, The Messenger, 126, 41

Faherty, J. K., Burgasser, A. J., Cruz, K. L., et al. 2009, AJ, 137, 1

Fritz, T., Gillessen, S., Trippe, S., et al. 2010, MNRAS, 401, 1177

Fűrész, G., Hartmann, L. W., Megeath, S. T., Szentgyorgyi, A. H., \& Hamden, E. T. 2008, ApJ, 676, 1109

Geballe, T. R., Knapp, G. R., Leggett, S. K., et al. 2002, ApJ, 564, 466

Goldman, B., Marsat, S., Henning, T., Clemens, C., \& Greiner, J. 2010, MNRAS, 405,1140

Golimowski, D. A., Leggett, S. K., Marley, M. S., et al. 2004, AJ, 127, 3516

González Hernández, J. I., Caballero, J. A., Rebolo, R., et al. 2008, A\&A, 490, 1135

Hambly, N. C., Collins, R. S., Cross, N. J. G., et al. 2008, MNRAS, 384, 637

Herbst, T. M., Thompson, D., Fockenbrock, R., Rix, H., \& Beckwith, S. V. W. 1999, ApJ, 526, L17

Hernández, J., Hartmann, L., Megeath, T., et al. 2007, ApJ, 662, 1067

Hewett, P. C., Warren, S. J., Leggett, S. K., \& Hodgkin, S. T. 2006, MNRAS, 367,454
Hodapp, K. W., Iserlohe, C., Stecklum, B., \& Krabbe, A. 2009, ApJ, 701, L100 Jeffries, R. D., Maxted, P. F. L., Oliveira, J. M., \& Naylor, T. 2006, MNRAS, 371, L6

Knapp, G. R., Leggett, S. K., Fan, X., et al. 2004, AJ, 127, 3553

Lawrence, A., Warren, S. J., Almaini, O., et al. 2007, MNRAS, 379, 1599

Lee, H., Chen, W. P., Zhang, Z., \& Hu, J. 2005, ApJ, 624, 808

Lodieu, N., Zapatero Osorio, M. R., Rebolo, R., Martín, E. L., \& Hambly, N. C. 2009, A\&A, 505, 1115

Lucas, P. W., \& Roche, P. F. 2000, MNRAS, 314, 858

Lucas, P. W., Weights, D. J., Roche, P. F., \& Riddick, F. C. 2006, MNRAS, 373, L60

Luhman, K. L., Hernández, J., Downes, J. J., Hartmann, L., \& Briceño, C. 2008, ApJ, 688, 362

Marsh, K. A., Kirkpatrick, J. D., \& Plavchan, P. 2010, ApJ, 709, L158

McLean, I. S., McGovern, M. R., Burgasser, A. J., et al. 2003, ApJ, 596, 561

Metchev, S. A., Kirkpatrick, J. D., Berriman, G. B., \& Looper, D. 2008, ApJ, 676, 1281

Perryman, M. A. C., Lindegren, L., Kovalevsky, J., et al. 1997, A\&A, 323, L49 Pirard, J., Kissler-Patig, M., Moorwood, A., et al. 2004, in SPIE Conf. 5492, ed. A. F. M. Moorwood, \& M. Iye, 1763

Rayner, J. T., Cushing, M. C., \& Vacca, W. D. 2009, ApJS, 185, 289

Reylé, C., Delorme, P., Willott, C. J., et al. 2010, A\&A, 522, A112

Rosenthal, E. D., Gurwell, M. A., \& Ho, P. T. P. 1996, Nature, 384, 243

Sacco, G. G., Franciosini, E., Randich, S., \& Pallavicini, R. 2008, A\&A, 488, 167

Saumon, D., Hubbard, W. B., Burrows, A., et al. 1996, ApJ, 460, 993

Scholz, A., \& Jayawardhana, R. 2008, ApJ, 672, L49

Sherry, W. H., Walter, F. M., \& Wolk, S. J. 2004, AJ, 128, 2316

Sherry, W. H., Walter, F. M., Wolk, S. J., \& Adams, N. R. 2008, AJ, 135, 1616

Skrutskie, M. F., Cutri, R. M., Stiening, R., et al. 2006, AJ, 131, 1163

Smith, J. A., Tucker, D. L., Kent, S., et al. 2002, AJ, 123, 2121

Spiegel, D. S., Burrows, A., \& Milsom, J. A. 2011, ApJ, 727, 57

Tinney, C. G., Burgasser, A. J., Kirkpatrick, J. D., \& McElwain, M. W. 2005, AJ, 130, 2326

Zapatero Osorio, M. R., Béjar, V. J. S., Martín, E. L., et al. 2000, Science, 290, 103

Zapatero Osorio, M. R., Béjar, V. J. S., Martín, E. L., et al. 2002, ApJ, 578, 536

Zapatero Osorio, M. R., Béjar, V. J. S., Bihain, G., et al. 2008, A\&A, 477, 895 